\title{
MeMLO: Mobility-Enabled Multi-Level Optimization Sensor Network
}

\author{
M.C. Rajalakshmi ${ }^{1}$, A.P. Gnana Prakash ${ }^{2}$ \\ ${ }^{1}$ University of Mysore, Mysore, India \\ ${ }^{2}$ Deptof Studies in Physics, Univewrsity of Mysore, Mysore, India
}

\section{Article Info}

\section{Article history:}

Received Jun 13, 2016

Revised Jan 20, 2017

Accepted Jan 30, 2017

\section{Keyword:}

Battery

Clustering

Network lifetime

Optimization

Optimization

Wireless sensor network

\begin{abstract}
The paper presents a technique called as Mobility-enabled Multi Level Optimization (MeMLO) that addressing the existing problem of clustering in wireless sensor net-work (WSN). The technique enables selection of aggregator node based on multiple optimi-zation attribute which gives better decision capability to the clustering mechanism by choosing the best aggregator node. The outcome of the study shows MeMLO is highly capable of minimizing the halt time of mobile node that significantly lowers the transmit power of aggregator node. The simulation outcome shows negligible computational com-plexity, faster response time, and highly energy efficient for large scale WSN for longer simulation rounds as compared to conventional LEACH algorithm.
\end{abstract}

Copyright $(0) 2017$ Institute of Advanced Engineering and Science. All rights reserved.

Corresponding Author:

M.C Rajalakshmi, University of Mysore, India. E-Mail: rajhar70@gmail.com

\section{INTRODUCTION}

With the increasing adoption of WSN in upcoming application like Internet-of-Things, the awareness of the potential features and limitations are being consistently a point of focus among the research community. A sensor node is a very miniature device that can sense environmental events and uses routing principle to transmit the data to base station. As sensors are deployed in unmanned surveilled area, so it is essential that such sensor should optimally operate even in adverse conditions too. In order to support such operation, a sensor should have enough battery lifetimes, which is one of the restricted resources in them. Apart from this, there are many reasons of energy depletions. The prominent reason is the process called as data aggregation which requires a specific level of transmittance energy for each sensor node to forward the data. Hence, there are various studies [1-2] that have focused on energy efficient clustering process for enhancing the network lifetime. However, there are major pitfalls too in this. The secondary reason is routing protocol in WSN. Among different forms of protocol i.e. flat, hierarchical, and hybrid [3], availability of standard energy efficient routing protocol is extremely less compared to other routing techniques in WSN. Among the energy efficient routing technique, LEACH protocol [4] is one of the most frequently used approach for benchmarking in $95 \%$ of the research work. The prime reason behind this is LEACH is one of the protocols that was tested on original MicaZ motes and has potential supportability of hardware circuitry of the motes along with mathematical formulation for antenna theory. LEACH was tested both on hardware as well as on computational based approach and hence its reliability score is top most compared to other energy efficient protocols e.g. PEGASIS [5], TEEN [6], APTEEN [7], HEED [8], that were tested originally from hypothetical or simulation viewpoint. Although, LEACH has many new variants but still none of the variants of LEACH was testified on real-time motes [4]. However, LEACH too suffers from various pitfalls. This paper presents a technique of energy efficiency and multi level optimization for enhancing the network lifetime considering the mobility. Section 1.1 discusses about the background followed by Section 1.2 that 
discusses proposed system. Research methodology is discussed in Section 2 followed by Algorithm implementation in Section 3. Result discussion is carried out in Section 4 followed by summary of this paper in Section 5.

\subsection{Background}

This section brief the studies being carried out towards addressing energy problems in wireless sensor network. Our prior study has already reviewed existing techniques for addressing power issues [9]. However, this section will present more updates on recent work being carried out and limitation associated with it.

Adnan et al. [10] have adopted bio-inspired algorithm for performing clustering in wireless sensor network. Although the outcome of the technique showed better energy efficiency compared to LEACH, but the limitation of the study is its focused on small scaled network and the nodes could hardly sustain around 800 rounds of simulation. Another limitation of the study is that it is not applicable to multi-hop networks. Study on similar direction of using bio-inspired techniques has been carried out by Seelam et al. [11] where the authors have perform optimization using BAT algorithm. The experimental results shows better throughput, reduced delay, reduced retransmission attempts, and minimal data drop. The limitation of these techniques is its less focus on energy efficiency and more focus on communication performance. However, discussing communication performance with energy factor is not highly convincing as sensors works on radio-energy model. A recent work by Pei et al. [12] has performed an enhancement of conventional LEACH algorithm tested on sensor network equipped with cognitive radio systems. The paper has uniquely presented its mechanism to address the dynamics of sensor network topology that relates to energy consumption for multi-hop transmission. However, the mechanism selects cluster head based on energy factor for which reason the nodes cannot sustain more than 800 simulations round. Although, the outcome of the study was found better than existing techniques but outcome was witnessed with steep fall of residual power after $800^{\text {th }}$ round, which is limitation for mission critical application in sensor network. Study on similar direction was carried out by Udompongsuk [13] where the authors have introduced a statistical technique for selection of clusterhead. The presented technique uses moving average over energy dissipation by the clusterhead. The prime limitation of this technique is i) usage of moving average leads to more dependency on past routing data, ii) usage of moving average may also lead to outliers if heterogeneous network is considered. Such problems towards heterogeneous network were found addressed by Patil and Kulkarni [14]. The authors have presented a dual layer of heterogeneity for computing energy dissipation along with consideration of Received Signal Strength Indicator. Although, the outcome shows better efficiency but the technique doesn't have any forms of optimization, for which reason it can be quite computational expensive in nature. Gautam et al. [15] have presented another clustering technique that uses Voronoi diagram as well as Ant system. The limitation of the study is that the outcomes were not compared with energy efficient protocols in wireless sensor network and the approach is more deterministic in nature and hence it has less chances of optimization in future.

Meenakshi and Kumar [16] have carried out the study towards energy efficiency by enhancing the conventional LEACH algorithm. Inspite of superior outcomes, the algorithm suffers from some critical limitation i.e. localizing base station at the center, which is quite impractical. Neamatollahi et al. [17] have considered multiple criteria for formulating cluster in wireless sensor network. The technique limits its usage in homogenous network only. Exactly similar forms of study was also carried out by Wei et al. [18] where the technique presented a unique clustering mechanism to support multihop data communication. However, the study limits its selection process of clusterhead based on energy factor only. A unique technique of unequal clustering process was presented by $\mathrm{Yu}$ et al. [19] that balance the energy required for unequal clustering process in wireless sensor network. The study outcome shows better energy efficiency charecteristics but limits from its applicability in small scale network only. Zhu et al. [20] have presented a unique clustering mechanism for enhancing the network lifetime using Hausdorff distance-based clustering process. The prime limitation of the study is that its outcome was not compared with energy efficient techniques.

Hence, it can be seen that there are some major research work has been carried out towards energy conservation problem in sensor network where majority of the techniques are based on clustering. Although, the prior techniques creates a good based for upcoming study but problem still existing in the area of energy efficient optimization approach, which is found quite in less amount in existing research work. The next section outlines the contribution of the proposed study that attempts to bridge the research gap of energy efficient optimization in wireless sensor network for enhancing the network lifetime. 


\subsection{The Proposed Model}

The prime purpose of the proposed study is to present a framework that has the capability to implement superior degree of multi level optimization in wireless sensor network for the purpose of enhancing the network lifetime. The prime contributions of the proposed system are as follows:

a. To formulate environment for multi-level optimization for enhancing network lifetime

a) To introduce a novel attribute called as Primary Optimization Attribute that performs filtering of candidate nodes based on location, distance, residual energy, and neighbor nodes.

b) To introduce a novel attribute called as Secondary Optimization Attribute that considers relative distance factors.

b. To perform selection of an aggregator node based on primary and secondary optimization attribute.

c. To formulate a condition of mobility of sink that collects aggregated data from aggregator node in particular defined areas.

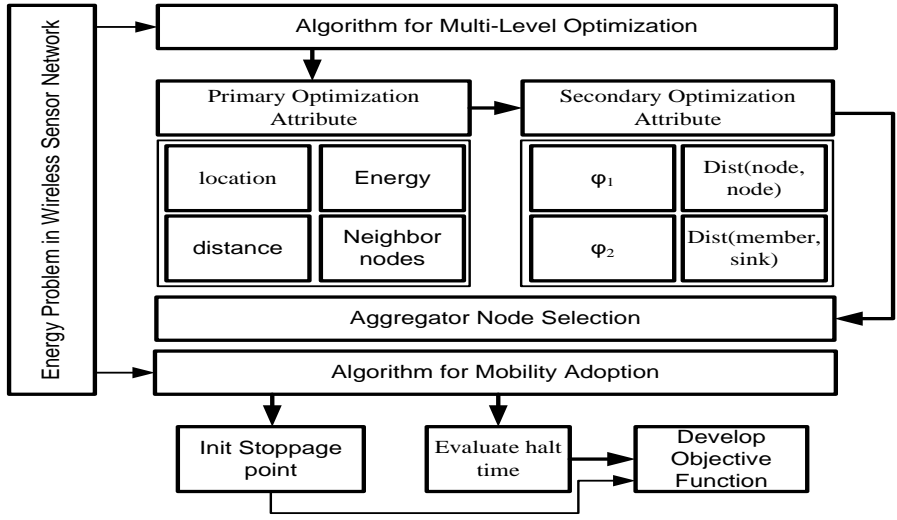

Figure 1. Architecture of MeMLO

Figure 1 shows the architecture of proposed MeMLO which addresses the problems of energy dissipation in wireless sensor network. The proposed MeMLO is a continuation of our prior techniques [21-22]. The next section discusses about the research methodology adopted for developing MeMLO.

\section{RESEARCH METHODOLOGY}

The design and development of our prior MLO algorithm [22] was total based on analytical background. The target was to use the optimization potential of MLO algorithm for accomplishing an enhanced network lifetime with efficient clustering mechanism. The technique also considers input parameter with a probability that a sensor can become a aggregator node during consecutive cycle of data aggregation. Even our prior work of Empirical Modelling of Energy Optimization EO-RTD [21] also is more focused on clustering process, so it will also emphasize on the selection process of the core object, which is an aggregator node. The development of the algorithm of the proposed system is done in analytical approach to ensure better evidential ground.

\subsection{Reason for Adoption}

The design principle of our prior work EO-RTD [21] allows breaking the myth that a cluster head should be only selected based on residual energy. The technique presented in EO-RTD [21] also provides two conditions of clustering process i.e. where both the conditions allows assessing multiple parameters e.g. location, energy, neighborhood, referential position, aggregator node-sink node distance, and different aggregator node distance. The study outcome also exhibits better network lifetime and hence both EO-RTD [21] and MLO [22] is an energy efficient clustering process.

\subsection{Limitation}

However, our prior model EO-RTD [21] and MLO [22] has not been tested over mobility aspect. As many of the upcoming applications and research over wireless sensor network is considered based on 
mobility, so our prior techniques may find challenge in such environment to operate effectively causing further degradation of network lifetime. Figure 2 shows chema used in Proposed Study

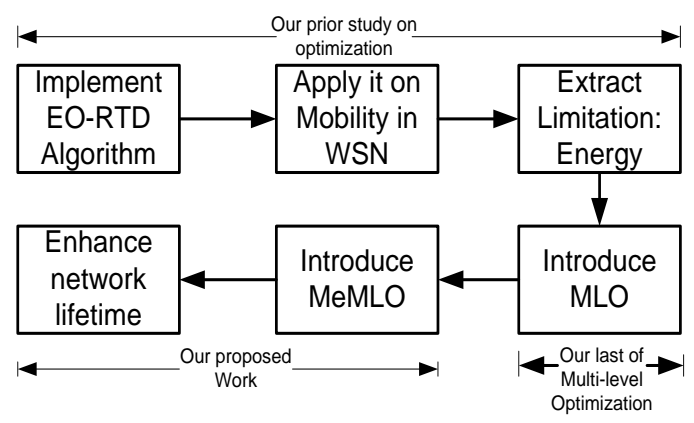

Figure 2. Schema used in Proposed Study

\subsection{Methodology for MeMLO}

A careful observation of our prior techniques will shows that it has enhanced network lifetime to a large extent; however, it is limited to static nodes which will be less practical for futuristic application where nodes are studied with mobility. Hence, the network lifetime and clustering operation introduced by our prior techniques is now subjected for multi level optimization considering a single mobile sink defined for one simulation area. It is meant for performing Mobility-enabled Multi-level Optimization (MeMLO) where rate of data aggregation can be speeded up along with lesser consumption of energy. Hence the proposed system is the enhanced version of our prior optimized techniques in WSN by incorporating mobility as well as QoS efficient power factor. Following are the contributory points of proposed system:

a. Assumption: MeMLO considers presence of a single mobile node (i.e. sink), which stops at particular defined location within simulation area called as stoppage, where aggregated data are transmitted from clusterhead to mobile sink.

b. Optimization Theory: The incorporated optimization theory will be finding the objective function that can minimize the halt time (It is a time taken for a mobile sink to be in its defined stoppage area).

The design and development of MeMLO mainly consists of a $i$ ) communication model, $i$ ) clustering process, iii) Selection of Clusterhead, and iv) mobility aspect of sink. The design of the communication model is carried out with respect to the first order radio energy model. The study considers the mobility only with respect to sink and other nodes doesn't move. The design principle assumes that orientation of the sink is carried out equally in equal interval of time on $n$ number of location, which can be again customized by the user. Customization will mean that for small area or for slower traffic, $\mathrm{n}$ could be limited to 1-2 location called as stoppage. The duration spend by the nodes in the stoppage is called as halt time, within which aggregated data is transmitted to sink from active clusterhead. The formation of clustering is quite simple in MeMLO.
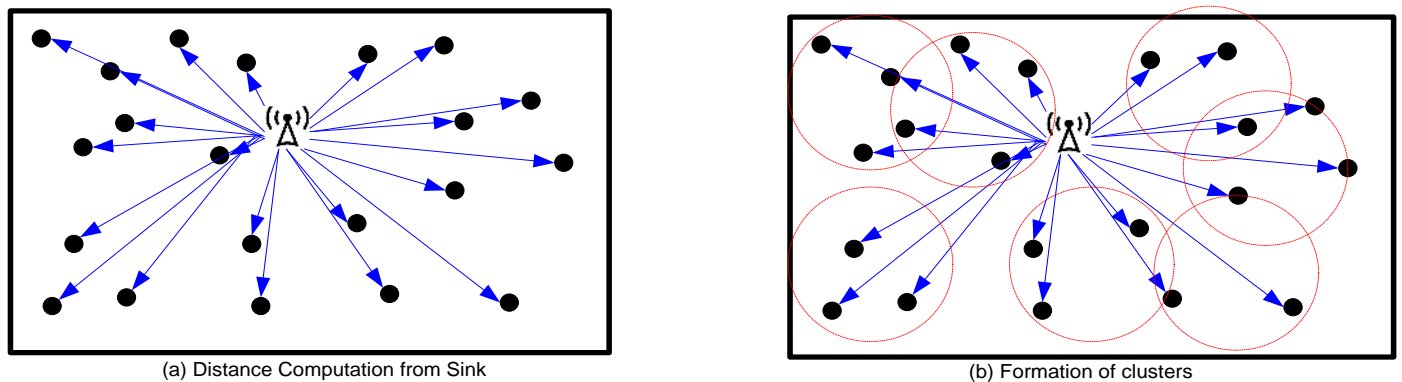

Figure 3. MeMLO Cluster Formation

Initially, the sink transmits a beacon to its adjacent sensors in order to receive all the distinct information of other nodes that assists the sink node to compute the sensor located at greater distance. It does 
so by comparing all the individual distance with other sensors. In this clustering mechanism, the cluster initiates formulation only from the node located at greater distance which is very far from the sink. This is done intentionally in order to extend the coverage area of sink to maximum possibility. This operation also ensures minimization of partitioned nodes if residing within simulation area. Similar process is repeated to formulate many numbers of clusters. Based on MeMLO model, it also selects the neighbor density along with the distance to ensure that cluster is formulated effectively with the presence of nodes. Figure 3 exhibits the distance computation from sink followed by formation of clusters. A closer look into the clustering process will also show that node-density as well as distance factor assists in the formation of the clusters. Once the cluster is formulated the next step is to perform selection of the clusterhead. In this case, a cluster makes the selection of the clusterhead on the basis of a cost function $c$ that is assigned on all the sensors in simulation area. The system performs computation of cost for all the sensors and based on the sensors with higher value of cost is considered to be clusterhead. In proposed system, cost $c$ can be defined as a matrix consisting of elements information from both single and multi scale clustering approximation process. It will mean that cost is a metric that has information about location, energy, neighborhood, referential localization, aggregator node -sink node distance, and inter cluster head distance. Hence, more the size of information for one sensor node, the probability of getting it elected as aggregator node is also more. However, this computation can lead to selection of multiple nodes as aggregator node in one region. Hence in order to avoid it, all the nodes with higher value of cost are also checked if they have also higher value of new cost factor calculated by residual energy divided by distance between the sink and a sensor. The last stage is to implement the mobility of the sink. The design principle considers particular location of stoppage in order to perform data aggregation by mobile sinks. It is to be noted that there is only one mobile sink.

\section{ALGORITHM IMPLEMENTATION}

The development of the proposed MeMLO technique is carried out in Matlab with 500-1000 sensor nodes distributed randomly over $1000 \times 1200 \mathrm{~m}^{2}$. The simulation time is kept at 200 seconds with 0.5 as path loss exponent. The simulation analysis was carried over both constant as well as variable bit rate traffic with IEEE 802.11 as standard MAC model. The system considers channel capacity of $250 \mathrm{kbps}$ and 0.2 seconds as channel sensing time. Data packet is considered for size of 2000 bytes with mobility of sink initialized as 50 $\mathrm{m} / \mathrm{s}$. The energy parameters considered for evaluating energy dissipation are initialized as 0.5 Joule as transmission energy, 0.25 Joule as receiving energy consumption, and 0.035 Joule as ideal mode of power consumption.

The description of the algorithm is as discussed below:

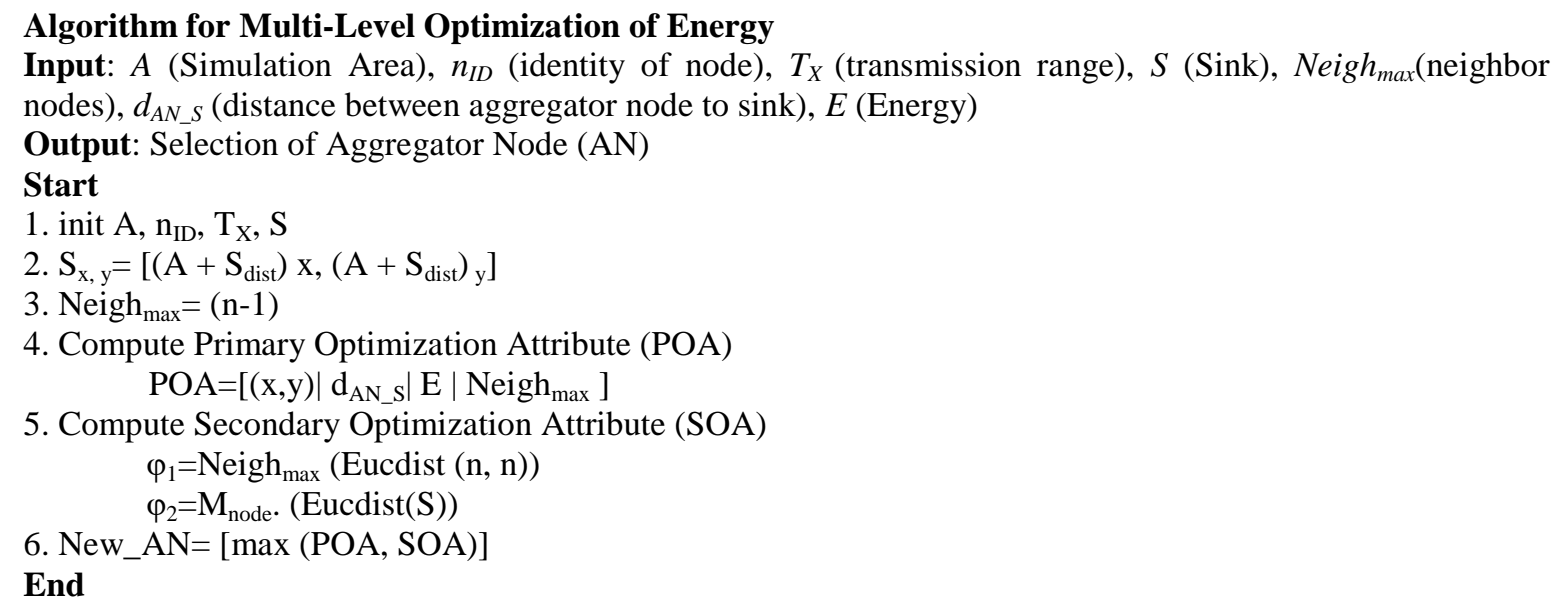

The above mentioned algorithm takes the input of simulation area, number of nodes, transmission range, and sinks position and implemented multi level optimization technique for enhancing the network lifetime. The optimization is carried out by considering both primary and secondary optimization attribute i.e. POA and SOA. The POA is an attribute that holds information about location of nodes, distance between aggregator node and sink node, remnant energy of node, and maximum neighbor nodes. The SOA is an attribute that holds two further matrices i.e. $\varphi_{1}$ and $\varphi_{2}$ which posses Euclidean distance between the interacting nodes and member node to sink respectively. The last step is to select the sensor with maximum value of both SOA and POA to be considered as an aggregator node. The algorithm mainly assists in 
implementing a novel optimization technique where selection of an aggregator node is done on the basis of multiple parameters apart from residual energy. The steps used in developing the algorithm are as follows:

a. Implement the conventional technique [21] and [22] for optimization algorithm that takes the input argument of battery and neighbor density for calculating the probability.

b. The algorithm then checks for presence of any aggregator node during the present simulation round.

c. The technique of MeMLO supports two level of optimization techniques i.e. i) Primary Optimization Attribute (POA) and ii) Secondary Optimization Attribute (SOA).

a) POA performs selection of the aggregator node based on location, energy, and neighborhood

b) SOA performs selection of aggregator node based on reference location, aggregator node and sink node distance, and distance from member node to sink.

d. Based on the maximum values of POA and SOA, an aggregator node is selected, which continues the progress of data aggregation process.

e. Under this process, the proposed system significantly overcomes the clustering problems of LEACH and enhances to multi-level optimization techniques.

\section{Algorithm of Mobility Adoption}

Input: $n$ (number of nodes), node_loc (node localization), sink_loc (sink localization), $c$ (cost), $E_{\text {res }}$ (residual energy), $d_{n \_s}$ (distance from node to sink), $h_{t}$ (halt time)

Output: pkt (data packet)

\section{Start}

1. Init $\mathrm{n}$, node_loc, sink_loc, pkt, ht

2. Estimate $c=E_{r e s} / d_{n \_s}$.

3. Evaluate halt time

$$
H_{t}=\int_{x=1}^{n_{H}} h_{t}^{x} d x
$$

4. Evaluate Objective function

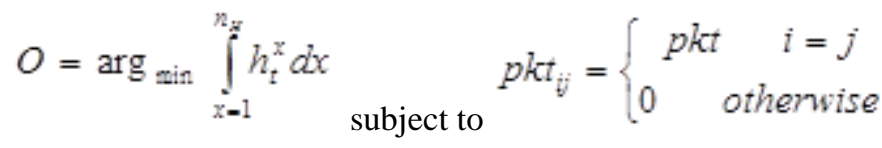

End

The prime purpose of proposed system is to provide significant level of optimization for the prior algorithm by incorporating more features of optimized clustering. The study brings more novelty by considering a presence of a mobile sink which has infinite availability of resources and the most interesting part of the study is use the feature of mobility of sink for enhancing the clustering approximation theory presented by multi-level optimization. The study considers a uniform trajectory of a sink node from one to another cluster in WSN. The algorithm considers that a sink can stop in $\mathrm{nH}$ number of halt point or so called stoppages within simulation area. The coordinates of the stoppages are predefined. The algorithm work as following:

The algorithm initially computes the profile of halt time.

a. Depending on the halt time, the algorithm initiates its tour towards the stoppages.

b. The algorithm the computes the cumulative time of journey to complete one simulation round of data aggregation.

c. By doing this, the proposed system exhibits two features:

a) Advantage: i) Due to multiple stoppage, the rate of data aggregation increases, ii) lesser delay in packet transmission, iii) significantly lowing of energy dissipation, iv) sink is free from energy computation so energy-based calculations are completely free from it.

b) Disadvantage: Due to multiple stoppages, halt time as well as journey time of mobile sink may increase.

c) Incorporated Solution to address this problem: Developed an objective function $O$ that can significantly minimize the halt time

d. Transmission of data $p k t$ increased on multiple rounds and significantly enhanced network lifetime. 
Hence, it can be seen that proposed technique implements multiple level of optimization and is capable of minimizing the energy consumption even considering the mobility of the sink node. The direct impact of this scheme can results in better transmission rate as well as highly optimized network lifetime. The memory consumption of the algorithm is not more than 9-11 bytes for one round of data aggregation and response time is approximately 0.2 seconds on normal computing environment. The next section discusses about the result discussion.

\section{RESULT AND DISCUSSION}

This section discusses about the outcomes accomplished from the proposed study with respect to individual outcomes and comparative outcomes.

\subsection{Individual Outcomes}

The individual outcomes of the study are assessed with respect to residual energy, alive nodes, and dead nodes. Figure 4 shows that residual energy for the proposed study degrades down till $2000^{\text {th }}$ simulation rounds and then starts depleting its energy.

However, residual energy may not be the only parameter to judge the affectivity in clustering with respect to clustering. Hence, the proposed studies consider evaluating number of alive and dead nodes for 5000 simulation rounds. The outcomes are highly positive to find proposed technique shows better availability of alive nodes till $2000^{\text {th }}$ simulation rounds, which starts stiff degrading from $4000^{\text {th }}$ simulation round onwards as shown in first graphical outcome in Figure 4 and Figure 5. Similarly, the outcomes of the dead nodes per rounds shows increase of dead nodes in $4000^{\text {th }}$ simulation rounds. Hence, the study outcomes shows better optimized network lifetime.

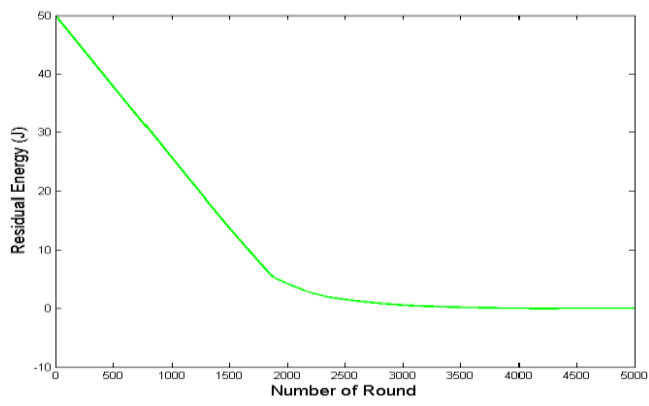

Figure 4. Outcome of Residual Energy for MeMLO

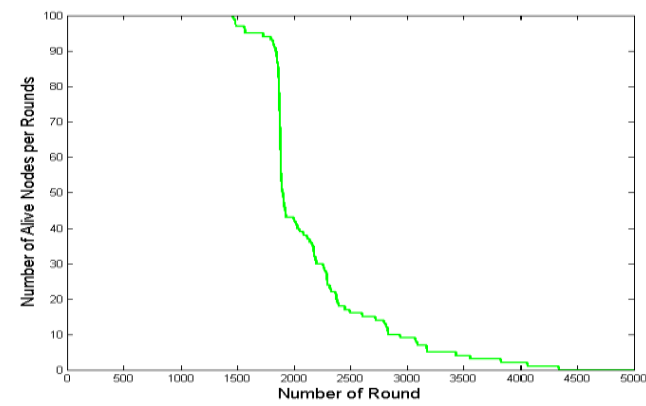

Figure 5. Outcome of Alive Nodes for MeMLO

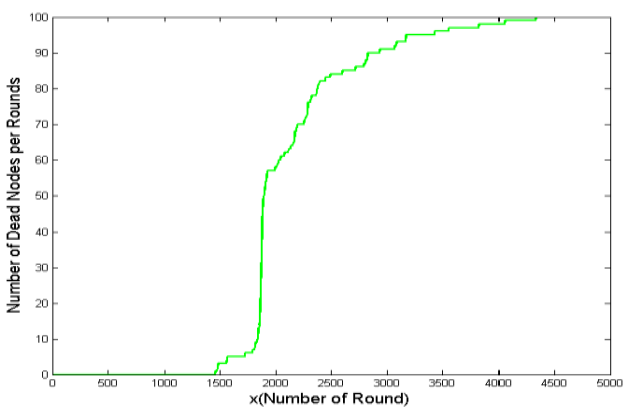

Figure 6. Outcome of Number of Dead Nodes per Rounds in MeMLO

\subsection{Comparative Outcomes}

The outcome of the proposed study is compared with the standard LEACH algorithm to assess its energy effectiveness with respect to

The study outcome in Figure 7, 8, 9 clearly shows that proposed study provides a better mechanism of clustering whose direct result can be seen in the energy efficiency. The proposed system has better residual 
energy conservation, more number of alive nodes of increasing rounds and exhibits highly minimized energy fluctuation in comparison to standard energy efficient algorithm. LEACH was never meant for supporting multihop communication system with higher dependency of sink to be located in center of simulation. Hence, LEACH has serious design issues although it is one of the standard energy efficient hierarchical algorithms till date. The proposed technique of MeMLO has higher degree of multi-level optimization involved in the design principle that results in more flexibility in clustering process. The network lifetime is also found to be significantly enhanced for 5000-7000 simulation rounds as compared to the existing optimization techniques till date on energy efficiency in wireless sensor network.

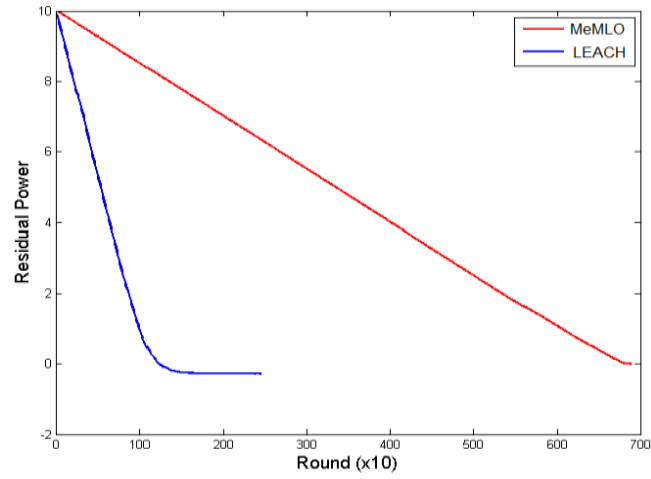

Figure 7. Comparative Outcome of Residual Energy for MeMLO

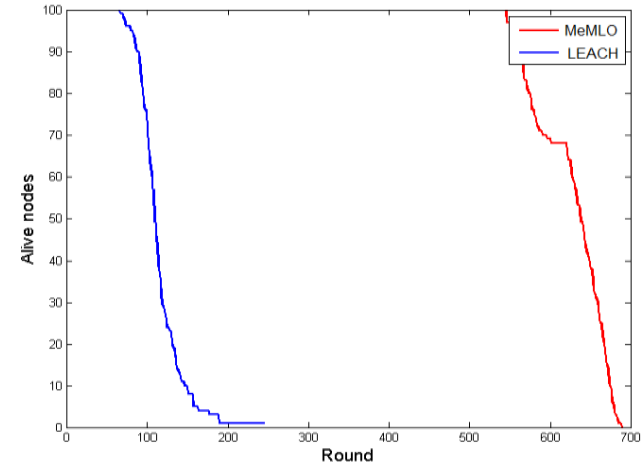

Figure 8. Comparative Outcome of Residual Energy for MeMLO

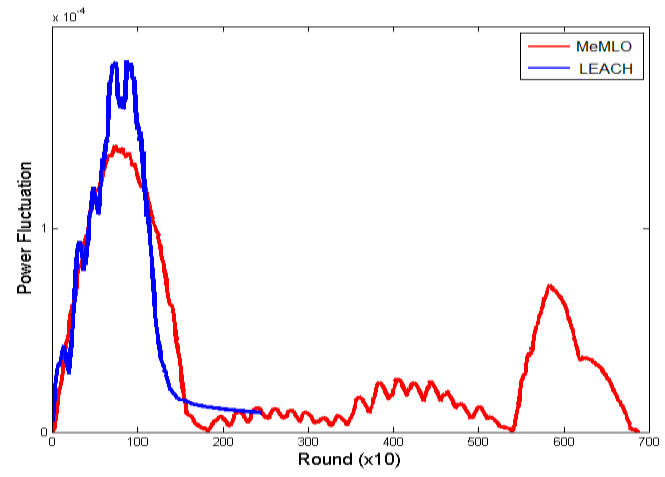

Figure 9. Comparative Outcome of Alive \& Energy Fluctuation in MeMLO

\section{CONCLUSION}

Energy is always the scarce resource in wireless sensor network and clustering is the most frequently adopted technique to ensure the longevity of the sensor nodes. Till last decades there have been massive literatures towards addressing energy efficient clustering mechanism. Out of such standards research contribution, some techniques have received a significant recognition and are widely studied in relation to energy efficient clustering. But, such existing standards suffers from i) lacks of broader scope of optimization, ii) usage of too much complex algorithm that affects communication performance and longevity of nodes, and iii) availability of lesser number of energy efficient clustering standards. The biggest pitfalls of existing clustering mechanism is its impractical assumptions i.e. i) selection of sink on the basis of residual energy only, ii) localizing the base station in the center of simulation (position dependency), iii) lack of supportability of multihop communication in energy efficient hierarchical protocols. Hence, this paper addresses all such significant issues by presenting a novel clustering technique that performs potential optimization. Multiple criteria have been formulated for selection of aggregator node, which gives better edge to energy conservation. Using first order radio model, the energy assessment shows that proposed cluster optimization technique ensure optimal longevity of a sensor node. 


\section{REFERENCES}

[1] G. Vennira Selvi, R. Manoharan, "A Survey of Energy Efficient Unequal Clustering Algorithms for Wireless Sensor Networks", International Journal of Computer Applications, vol. 2, 2013

[2] V. Kumar, M. Bansal, M.K. Rai, "Literature Survey on Energy Efficient Clustering and Routing in Wireless Sensor Networks", International Journal of Computer Application, Volume 5, No. 3, April 2015

[3] A. Anwar, D. Sridharan, "A Survey on Routing Protocols for Wireless Sensor Networks in Various Environments", International Journal of Computer Applications, Vol. 112, No. 5, February 2015

[4] W. Heinzelman, A. Chandrakasan, and H. Balakrishnan, "Energy-Efficient Communication Protocols for Wireless Microsensor Networks", Proceedings of the 33rd Hawaaian International Conference on Systems Science (HICSS), January 2000

[5] S. Lindsey, and C. Raghavendra, "PEGASIS: Power- efficient gathering in sensor information systems", IEEE Aerospace Conference Proceedings, 2002, pp. 1125-1130.

[6] A. Manjeshwar and D.P. Agarwal, "TEEN: a routing protocol for enhanced efficiency in wireless sensor networks", 1st International Workshop on Parallel and Distributed Computing Issues in Wireless Networks and Mobile Computing, April 2001.

[7] A. Manjeshwar and D.P. Agarwal, "APTEEN: A hybrid protocol for efficient routing and comprehensive information retrieval in wireless sensor networks", Parallel and Distributed Processing Symposium, Proceedings International, IPDPS 2002, pp. 195-202.

[8] O. Younis and S. Fahmy, "Distributed Clustering in Ad-hoc Sensor Networks: A Hybrid, Energy-Efficient Approach", In Proceedings of IEEE INFOCOM, Hong Kong, an extended version appeared in IEEE Transactions on Mobile Computing, vol. 3, Iss.4, 2004

[9] M.C. Rajalakshmi. "Review of Typical Power Conservation Techniques in Wireless Sensor Network". International Journal of Computer Applications, vol. 88, No.10, pp.7-13, February 2014

[10] Md. A. Adnan, M.A. Razzaque, Md. A. Abedin, S.M.S. Reza and M.R. Hussein, "A Novel Cuckoo Search Based Clustering Algorithm for Wireless Sensor Networks", Springer Journal, Advanced Computer and Communication Engineering Technology, Lecture Notes in Electrical Engineering, 2016

[11] K. Seelam, M. Sailaja and T. Madhu, "An Improved BAT-Optimized Cluster-Based Routing for Wireless Sensor Networks", Springer Journal, Intelligent Computing and Applications, Advances in Intelligent Systems and Computing, 2015

[12] E. Pei, H. Han, Z. Sun, B. Shen and T. Zhang, "LEAUCH: low-energy adaptive uneven clustering hierarchy for cognitive radio sensor network", Springer- EURASIP Journal onWireless Communications and Networking, 2015

[13] K. Udompongsuk, C.S. In, C. Phaudphut, "MAP: An Optimized Energy-Efficient Cluster Header Selection Technique for Wireless Sensor Networks", Springer Journal, Advances in Computer Science and Its Applications, Lecture Notes in Electrical Engineering, 2014

[14] P.R. Patil and U.P. Kulkarni, "Energy-Efficient Cluster-Based Aggregation Protocol for Heterogeneous Wireless Sensor Networks", Springer-Journal, Intelligent Computing, Networking, and Informatics, Advances in Intelligent Systems and Computing, 2014

[15] N. Gautam, S. Sofat, and R. Vig, "An Ant Voronoi Based Clustering Approach for Wireless Sensor Networks", Springer Journals, Social Informatics and Telecommunications, 2014

[16] D. Meenakshi and S. Kumar, "Energy Efficient Hierarchical Clustering Routing Protocol for Wireless Sensor Networks", Springer Journal, Social Informatics and Telecommunications Engineering, pp.409-420, 2012

[17] P. Neamatollahi, H. Taheri, M. Naghibzadeh, "DESC: Distributed Energy Efficient Scheme to Cluster Wireless Sensor Networks", Springer Journal, International Federation for Information Processing, pp.234-246, 2011

[18] D. Wei, Y. Jin, S. Vural, K. Moessner, "An Energy-Efficient Clustering Solution for Wireless Sensor Networks", IEEE Transactions On Wireless Communications, vol. 10, no. 11, November 2011

[19] J. Yu, Y. QI, G. Wang, "An energy-driven unequal clustering protocol for heterogeneous wireless sensor networks", Springer Journal of Control Theory Application, 2011

[20] X. Zhu, L. Shen, and T-S Peter Yum, "Hausdorff Clustering and Minimum Energy Routing for Wireless Sensor Networks", IEEE Transactions On Vehicular Technology, vol. 58, no. 2, February 2009

[21] M.C. Rajalakshmi and A.P. Gnana Prakash, "Energy Optimization for Large Scale Wireless Sensor Network using Real-Time Dynamics", International Journal of Computer Applications 108(7):40-46, December 2014

[22] M.C.Rajalakshmi, A.P. Gnana Prakash, "MLO: Multi-level Optimization to Enhance the Network Lifetime in Large Scale WSN", Springer, Emerging Research in Computing, Information, Communication and Applications, pp 265-271, 2015 